\title{
Exponentially Rapid Decoherence of Quantum Chaotic Systems
}

\author{
Arjendu K. Pattanayak and Paul Brumer \\ Chemical Physics Theory Group, University of Toronto, Toronto, Ontario, Canada M5S $3 H 6$
}

(Received 21 July 1997)

\begin{abstract}
We use a recent result to show that the rate of loss of coherence of a quantum system increases with increasing system phase-space structure and that a chaotic quantal system in the semiclassical limit decoheres exponentially with rate $2 \lambda_{2}$, where $\lambda_{2}$ is a generalized Lyapunov exponent. As a result, for example, the dephasing time for classically chaotic systems goes to infinity logarithmically with the temperature, in accord with recent experimental results. [S0031-9007(97)04643-7]
\end{abstract}

PACS numbers: $05.45 .+\mathrm{b}, 03.65 . \mathrm{Sq}, 05.40 .+\mathrm{j}$

Decoherence [1,2] which is, in general, the loss of interference effects in a quantal system due to its interaction with the environment [3], is important in fields that rely on the maintenance of delicate quantal coherence, including the rapidly growing areas of coherent control [4], quantum computation [5], and charge transport in mesoscopic devices [6]. It also plays a central role in treatments of classical-quantum correspondence [1,2]. Preliminary studies have suggested that systems which are chaotic in the classical limit decohere rapidly [7-9]. However, the rate of this decoherence and its dependence on system properties is unclear. Here we derive the functional form for the rate of decoherence, provide an explicit link between the rate and the extent of the system's phasespace structure and demonstrate that decoherence occurs exponentially, with a rate determined by a generalized Lyapunov exponent, for quantum systems with a chaotic classical analog. In addition, we note that the decoherence rate is reduced for $\hbar$ sufficiently large, although in general the decoherence of a quantal system may be maximally rapid for a finite value of $\hbar$. Finally, we show that our results explain the recent experimental result [6] that the decoherence rate in mesoscopic devices saturates as the temperature $T \rightarrow 0$. All of these results follow from an analysis of decoherence in light of a recent approach [10] which connects the exponentially fast growth of structure in the distribution dynamics of chaotic systems to a generalized Lyapunov exponent $\lambda_{2}$ of the classical dynamics in phase space.

Consider the Wigner representation $\left(\equiv \rho^{W}\right)$ [11] of a quantal density matrix; it is suitably normalized $\left(\operatorname{Tr}\left[\rho^{W}\right]=1\right)$, but is in general not a "pure state" $\left(\operatorname{Tr}\left[\left(\rho^{W}\right)^{2}\right] \neq 1\right)$. The relevant decoherence equation for $\rho^{W}$, derived from the quantum system evolving under the potential $V(q)$ and coupled to an external environment through the position operator, is [1-3,7]

$$
\begin{aligned}
\frac{\partial \rho^{W}}{\partial t}= & \left\{H, \rho^{W}\right\}+\sum_{n \geq 1} \frac{\hbar^{2 n}(-1)^{n}}{2^{2 n}(2 n+1) !} \frac{\partial^{2 n+1}}{\partial q^{2 n+1}} V(q) \\
& \times \frac{\partial^{2 n+1}}{\partial p^{2 n+1}} \rho^{W}+D \frac{\partial^{2}}{\partial p^{2}} \rho^{W} .
\end{aligned}
$$

The first term on the right of this equation is the Poisson bracket or classical Liouville operator, which gener- ates the classical evolution for $\rho^{W}$; the terms in $\hbar$ add the quantal evolution, with the expansion being formally valid for a potential $V(q)$ analytic in $q$. The decoherence is contained in the term dependent on $D$; its precise form and parameter dependence rests upon assumptions about the form of the system-environment coupling and the spectrum of the environment. The standard form [1], for example, has $D=2 \gamma M k_{B} T$, where $M$ is the system mass, $T$ the temperature of the environment, $k_{B}$ is the Boltzmann constant, and $\gamma$ an unknown measure of the system-environment coupling. Different forms for $D$ may also obtain [2,12], although $D$ always depends monotonically on the coupling and the temperature of the environment. The particular form of the coupling $D \frac{\partial^{2}}{\partial p^{2}} \rho^{W}$ comes from the choice of coupling through position; coupling through momentum would give $D \frac{\partial^{2}}{\partial q^{2}} \rho^{W}$. For full generality it is appropriate to use $D \nabla^{2} \rho^{W}$, where $\nabla^{2}$ is the Laplacian operator in phase space. By using the relationship $\frac{d \hat{A}}{d t}=\frac{\partial \hat{A}}{\partial t}+\frac{1}{i \hbar}[\hat{A}, \hat{H}]$ for the time evolution of an arbitrary operator $\hat{A}$, Eq. (1) can also be written as

$$
\frac{d \rho^{W}}{d t}=D \nabla^{2} \rho^{W} .
$$

We use the $\nabla^{2}$ form hereafter; the arguments are entirely unchanged if either the momentum or the position coupling is used.

Our criterion for monitoring the degree of system decoherence is the Renyi entropy $[1,13] S=\ln \left(\operatorname{Tr}\left[\left(\rho^{W}\right)^{2}\right]\right)$. This function maximizes at zero for a pure state and is a direct measure of the degree of mixing of the quantum state $[1,14]$. The time dependence of $S$ thus provides information about the rate at which a "coherent" quantum pure state is transformed into an "incoherent" or statistical mixture of states. It is straightforward to show, using Eq. (2), that

$$
\frac{d S}{d t}=2 D \frac{\operatorname{Tr}\left[\rho^{W} \nabla^{2} \rho^{W}\right]}{\operatorname{Tr}\left[\left(\rho^{W}\right)^{2}\right]} \equiv-2 D \chi^{2} .
$$

The quantity $\chi[10]$ (which is in general time dependent) affords insight insofar as it is the root-mean-square Fourier radius of the distribution, and a measure of phasespace structure. That is, if we Fourier expand any distribution $\rho$ as $\rho(p, q)=\iint d \mu d \nu e^{2 \pi i(\mu p+\nu q)} \tilde{\rho}(\mu, \nu)$, 
where the tilde now represents the Fourier-transformed function, we get that

$$
\begin{aligned}
\chi^{2} & =-\frac{\operatorname{Tr}\left[\rho \nabla^{2} \rho\right]}{\operatorname{Tr}\left[\rho^{2}\right]}=\frac{\operatorname{Tr}\left[|\nabla \rho|^{2}\right]}{\operatorname{Tr}\left[\rho^{2}\right]} \\
& =\frac{4 \pi^{2} \int d k k^{2}|\tilde{\rho}(k)|^{2}}{\int d k|\tilde{\rho}(k)|^{2}},
\end{aligned}
$$

where the second equality follows from integration by parts and where $k \equiv(\mu, \nu)$. Since higher Fourier modes are related to increased structure, $\chi$ measures the structure in the distribution, and Eq. (3) shows that the decoherence rate of a quantal system is directly proportional to the extent of system structure in phase space [15]. Note that since $\frac{d S}{d t}$ is negative definite, the system cannot "recohere" [16] and hence much of the decoherence is determined by the short-time behavior of $\chi(t)$. Therefore, as long as the term in $D$ is not initially dominant, we can make quantitative predictions of $d S / d t$ based upon the time dependence of the phase-space structure from the Hamiltonian part of the evolution for $\rho^{W}$ [17].

Consider first time-independent states (e.g., a mixture of Hamiltonian eigenstates). To first order, i.e., before decoherence becomes significant, we can estimate $\chi^{2}$ from the initial $\rho^{W}$, and find that lower energy states, with less structure, decohere more slowly. Thus, for example, for the $n$th eigenstate of the harmonic oscillator $\chi^{2} \propto n$ so that decoherence is proportional to $n$, explaining the numerical results of Knight and Garraway [18]. Hence we deduce that in general stationary distributions with lower energy are more stable with respect to environmental perturbation.

For time-evolving distributions we need to consider Eq. (3). For nonchaotic systems some insight results from a simple analysis since in this instance an adequate measure of the time-dependent $\chi^{2}$ is the time-averaged $\bar{\chi}^{2}$. For pure states of the harmonic oscillator, for example, working within the approximation that an initially pure state remains so, this criterion yields that minimum uncertainty coherent states are the most stable [1].

For chaotic systems insight obtains from examining the behavior of $\chi^{2}$ in the semiclassical limit. In this limit $\rho^{W}$ is known [10] to behave like the classical result, at least for early times. Consider then the behavior of $\chi^{2}$ for a classically chaotic system. Let the equations of motion of a point in phase space be $\dot{x}=f(x)$. The equations of motion for the vectors in the tangent space are obtained by linearizing the dynamics $x \rightarrow x+s$ around a fiducial trajectory to yield

$$
\frac{d \varsigma}{d t}=\mathcal{M} \varsigma,
$$

where the Jacobian matrix $\mathcal{M}$ has the elements $M_{i j}=\frac{\partial f_{j}}{\partial x_{i}}$ at the point $x(t)$. It is straightforward to show [10] that

$$
\frac{d}{d t} \nabla \rho=-\mathcal{M} \nabla \rho
$$

that is, the equations for evolution of $\varsigma$ and $\nabla \rho$ are identical except for a minus sign, where $\nabla \rho$ is the phase- space gradient of the classical probability density $\rho$. This implies that the usual maximal Lyapunov exponent may be computed from the distribution gradient evaluated along a trajectory as

$$
\lambda(x)=\lim _{t \rightarrow \infty} \frac{1}{t} \ln (|\nabla \rho(x(t))|) .
$$

We now note that $\chi^{2}$ is the distribution average of this gradient. Thus, adapting the results of the standard thermodynamic approach for similar averages which use s instead of $\nabla \rho$ [13], we have [10] that

$$
\lim _{t \rightarrow \infty} \frac{1}{t} \ln (\chi)=\lambda_{2} \text {. }
$$

Here $\lambda_{2}$, which is independent of trajectories, is a generalized Lyapunov exponent related to the ordinary Lyapunov exponent by $\lambda_{2}=\lambda+\zeta$, where $\zeta \geq 0$ measures the local phase-space fluctuations in $\lambda$ (equivalent to a measure of the degree of intermittency in chaos [13]). The quantity $\lambda_{2}$ has the particularly simple interpretation [10] in that it measures the exponentially rapid increase of structure for a chaotically evolving distribution in a Hamiltonian system and is in this sense a fundamental exponent for chaos in distribution dynamics. From this treatment it is clear that $\chi(t)$ grows exponentially rapidly in a chaotic system as

$$
\chi^{2}(t)=\chi^{2}(0)\left[\exp \left(2 \lambda_{2} t\right)+C\right]
$$

where $C$ in general fluctuates or grows slower than exponentially with time.

Thus, substituting Eq. (9) in Eq. (3) we get that the initial decoherence rate of a quantal system in the semiclassical limit, to first order in $D$, is

$$
\frac{d S}{d t}=-2 D \chi^{2}(0)\left[\exp \left(2 \lambda_{2} t\right)+C\right],
$$

an equation which displays a direct correlation between decoherence rates and the generalized Lyapunov exponent of the underlying classical system.

Note that Eq. (10) implies that in the limit of chaotic semiclassical behavior, all time-dependent quantum states decohere exponentially rapidly and hence that there is only a marginal dependence on the initial distribution. This is in contrast to nonchaotic systems where, as noted above, the rate is strongly affected by the initial state.

We have numerically solved the discrete map version of Eq. (1), with the full $\nabla^{2}$ coupling to the environment, for a quantum chaotic cat map $[19,20]$, to explore the nature of $S(t)$ as a function of $\alpha$ (a scaled $\hbar[19,20]$ ). Results for $\alpha=10^{-1}$ to $10^{-5}$ are shown in Fig. 1, with $D$ chosen at a typical value of $10^{-6}$. Several features are evident: (a) in the limit of $\alpha \rightarrow 0, S$ decreases exponentially rapidly initially with a rate determined by $\lambda_{2}=\lambda=$ 0.9624 [21], consistent with Eq. (10), (b) the initial decoherence rate increases with $\alpha$ for small $\alpha$, with maximal falloff for an $\alpha \approx 0.001$, and (c) for $\alpha$ sufficiently large, the entropy decreases quite slowly. 


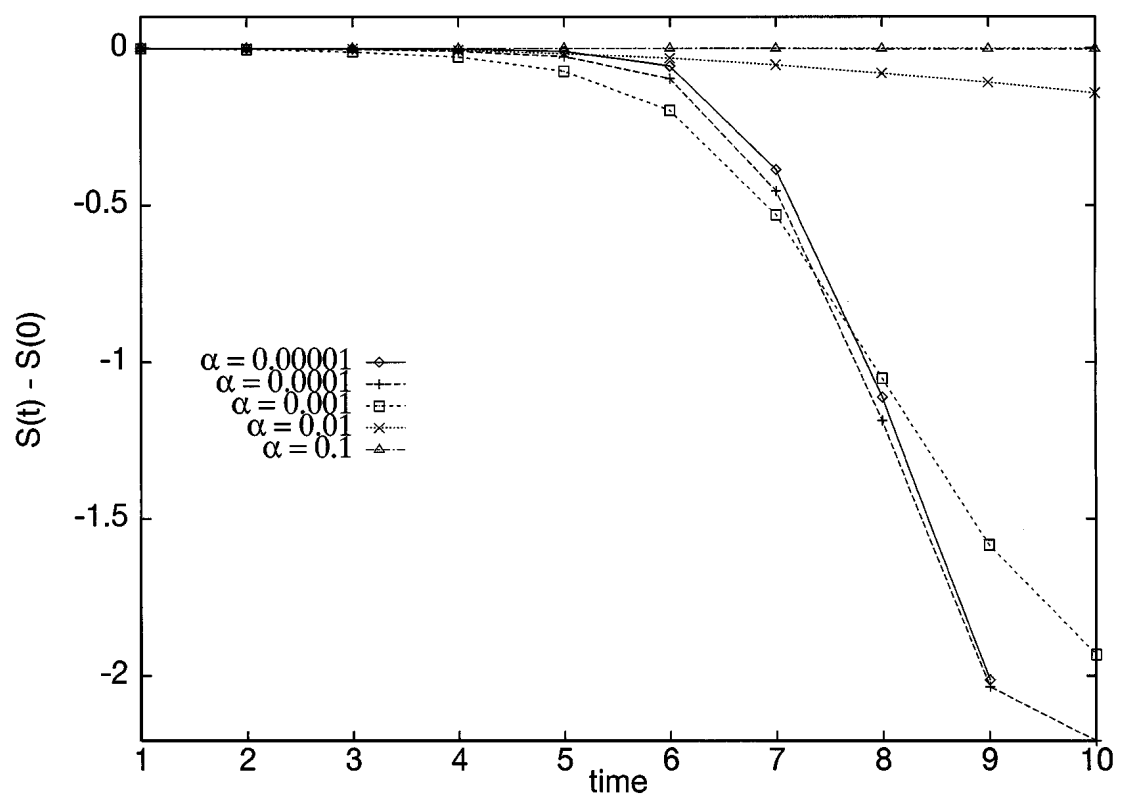

FIG. 1. Time dependence of the degree of incoherent mixing of quantal distributions as measured by the change in entropy $[S(t)-S(0)]$ for the chaotic quantal cat map coupled to the environment. The effect of approaching the classical limit is shown by varying $\alpha$ from $10^{-1}$ to $10^{-5}$, where $\alpha$ is a scaled $\hbar$. $D$, which is a measure of the coupling to the environment, is $10^{-6}$. See text for details.

Examination of the first quantum correction to Eq. (10) provides some insight into the observed dependence of $S(t)$ on $\alpha$. Note first that as $\hbar$ is increased away from 0 and for small $t$, there is an increase in the amount of structure in the distribution. That is, one expects $\rho^{W}$ to adiabatically follow the classical distribution, but with added fringes [22] (interference structures). The added structure can be seen, for example, in comparing the contour maps (Figs. 1-3, Ref. [20]) of the evolving distributions. From the first quantal term in Eq. (1), we can estimate that the quantum correction to an individual Fourier component $\tilde{\rho}(k)$ is $\tilde{\rho}(k) \rightarrow \tilde{\rho}^{W}(k) \approx \tilde{\rho}(k)(1+$ $\left.c^{\prime} \hbar^{2} k^{3}\right)$, where $c^{\prime}$ depends on the potential and includes all other constants. Thus, for an initial distribution that is not very structured (vanishing support at large $k$ ), $\rho^{W}$ is very close to $\rho$. However, even when the quantum terms are small, $\chi^{2}$ sums them over all the Fourier components, weighted by the factor $k^{2}$. A crude estimate [23] then yields that the quantum corrected $\chi_{q}^{2}$ relates to the classical estimate $\chi_{c}^{2}$ as $\chi_{q}^{2} \approx \chi_{c}^{2}\left(1+c \hbar^{2} \chi_{c}^{6}\right)$. Hence the quantum correction to $\chi^{2}$ can be quite large.

This analysis of the contribution of an individual Fourier component to the enhancement of structure breaks down for $\hbar^{2} k^{3}=\mathcal{O}(1)$; this happens rapidly, since for classically chaotic dynamics the support for the distribution at larger $k$ values increases exponentially fast. Hence, higher-order contributions also have to be estimated, which is in general extremely difficult. By this time, however, the second quantal effect enters: Quantum distributions resist the growth of structure at scales smaller than $\hbar$ [22] and the support of the distribution travels slowly across the $k \approx \frac{1}{\alpha}$ boundary in Fourier space. This is marked by [10] a slowdown of the growth of $\chi$ at $\mathcal{O}\left(\frac{1}{\alpha}\right)$. The interplay between these two effects leads to a value of $\hbar$ where the quantum system decoheres maximally rapidly for early times. Quantum systems for $\hbar$ larger than this critical value are, however, stable against decoherence, as seen in Fig. 1.

A second interesting effect emerging from this treatment is the dependence of the decoherence rate on $D$. It has been recently observed [6] that the decoherence rate for quantal mesoscopic devices goes to zero slower than any power of the temperature $T$ as the systems are cooled to near-zero $T$. The measure used in these experiments is the dephasing time, which is monitored through the change in coherent quantal backscattering effects in the device (weak localization) [6]. In our theoretical analysis, arguing that an increase in the degree of incoherent mixing reduces this coherent backscattering effect, a reasonable analog of the dephasing time may be taken to be the time $t_{R}$ at which the system has "sufficiently decohered," i.e., when the entropy has fallen to some fixed fraction of its initial value $R=S(0)-S\left(t_{R}\right)=\ln \left(\frac{\operatorname{Tr}\left\{\left[\rho^{W}(0)\right]^{2}\right\}}{\operatorname{Tr}\left\{\left\{\rho^{W}\left(t_{R}\right)\right]^{2}\right\}}\right)=1$, for example. Since these mesoscopic devices are disordered systems, with the electrons experiencing substantial chaotic scattering from impurities, Eq. (10) is expected to apply in the semiclassical limit. If we now solve for the effect on $S(t)$ of the dominant exponential term from this equation, we get the expression

$$
t_{R}=\frac{1}{2 \lambda_{2}} \ln \left[\frac{R \lambda_{2}}{D \chi^{2}(0)}+1\right] \text {. }
$$


The $1 / D$ term dominates as $T$, and hence $D, \rightarrow 0$ and indicates that, independent of the particular power-law dependence of $D$ on the temperature, the dephasing time goes to infinity logarithmically with vanishing temperature, i.e., slower than any power law. Equation (11) is, in fact, able to reproduce the general shape of Figs. 1 and 2 of Ref. [6], albeit with free parameters. In addition to this logarithmic dependence on $D$ in the semiclassical limit, a different effect shows up as the system becomes more quantal: Since (1) the decoherence rate grows as $D \chi^{2}$ and (2) the growth of structure slows down at $\chi=\mathcal{O}\left(\frac{1}{\hbar}\right)$, the decoherence term has a distinctly greater impact if $\sqrt{D} \geq \mathcal{O}(\hbar)$. This behavior can be seen in the slow decrease in $S$ for $\alpha=10^{-2}, 10^{-1}$ in Fig. 1.

In summary, we have demonstrated that the decoherence rate of a quantal system coupled to the environment is governed by the degree of structure in the system as measured by the quantity $\chi$. This has enabled us to make various deductions about the stability of distributions under the action of the environment which generalize several recent $[1,7,8]$ discussions. We have shown that since the structure of a distribution increases exponentially fast for a classically chaotic system, distributions in such systems decohere exponentially fast in the semiclassical limit, with the exponent given by the quantity $\lambda_{2}$. This behavior is manifest, for example, in the observation [6] that the dephasing time for semiclassical systems which are classical chaotic goes to infinity logarithmically with the temperature.

We thank the Natural Sciences and Engineering Research Council and the U.S. Office of Naval Research for support of this research. A. K. P. thanks Harold Baranger for useful discussions on Ref. [6] and Salman Habib for stimulating correspondence on an earlier version of this paper.

[1] W.H. Zurek, Phys. Rev. D 24, 1516 (1981); 26, 1862 (1982); Phys. Today 44, No. 10, 36 (1991); W. H. Zurek, S. Habib, and J. P. Paz, Phys. Rev. Lett. 70, 1187 (1993), and references therein.
[2] E. Joos and H. D. Zeh, Z. Phys. B 59, 223 (1985).

[3] A. O. Caldeira and A. J. Leggett, Physica (Amsterdam) 121A, 587 (1983), and references therein.

[4] For a recent review, see M. Shapiro and P. Brumer, J. Chem. Soc. Faraday Trans. 93, 1263 (1997).

[5] See, for instance, W. G. Unruh, Phys. Rev. A 51, 992 (1995).

[6] P. Mohanty, E. M. Q. Jariwala, and R. Webb, Phys. Rev. Lett. 78, 3366 (1997), and references therein.

[7] W. H. Zurek and J.P. Paz, Phys. Rev. Lett. 72, 2508 (1994).

[8] K. Shiokawa and B. L. Hu, Phys. Rev. E 52, 2497 (1995).

[9] A. Tameshtit and J. E. Sipe, Phys. Rev. A 47, 1697 (1993).

[10] A. K. Pattanayak and P. Brumer, Phys. Rev. E 56, 5174 (1997).

[11] See S. R. DeGroot and L.G. Suttorp, Foundations of Electrodynamics (North-Holland, Amsterdam, 1972) for an excellent review of the Wigner-Weyl technique.

[12] B. L. Hu, J.P. Paz, and Y. Zhang, Phys. Rev. D 45, 2843 (1992); 47, 1576 (1993).

[13] C. Beck and F. Schlögl, Thermodynamics of Chaotic Systems (Cambridge University Press, New York, 1993).

[14] X.-P. Jiang and P. Brumer, Chem. Phys. Lett. 208, 179 (1993).

[15] A different choice of coupling variable would lead to the decoherence rate being dependent on some combination of $\operatorname{Tr}\left[\rho^{W} \frac{\partial^{2}}{\partial p^{2}} \rho^{W}\right]$ and $\operatorname{Tr}\left[\rho^{W} \frac{\partial^{2}}{\partial q^{2}} \rho^{W}\right]$ in general.

[16] This is a direct manifestation of the irreversibility of Eq. (1), which is an approximation to the full dynamics (see, e.g., [3,1]).

[17] This is equivalently a first-order approximation in $D$ or a short-time result.

[18] P.L. Knight and B. M. Garraway in Quantum Dynamics of Simple Systems, edited by G.-L. Oppo, S. M. Barnett, E. Riis, and M. Wilkinson (Institute of Physics, Bristol, 1996).

[19] J. Wilkie and P. Brumer, Phys. Rev. E 49, 1968 (1994).

[20] A. K. Pattanayak and P. Brumer, Phys. Rev. Lett. 77, 59 (1996).

[21] In systems where the local stretching rate is constant, such as the cat map, $\lambda_{2}=\lambda$.

[22] M. V. Berry and N. L. Balazs, J. Phys. A 12, 625 (1979).

[23] A. K. Pattanayak and P. Brumer (unpublished). 